\title{
Investigación Guiada: Innovación Pedagógica Aplicada a la Enseñanza de Acústica
}

\author{
Guided Research: Pedagogical Innovation Applied to the Teaching of Acoustics
}

\author{
Sebastián P. Ferreyra ${ }^{\# * \$ 1}$, Gabriel A. Cravero ${ }^{\# \S}$, Ana M. Moreno ${ }^{\# *}$, José Y. Simes ${ }^{\#}$, Juan F. López ${ }^{\#}$

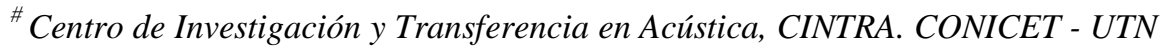 \\ Maestro López esq. Cruz Roja Argentina. Ciudad Universitaria Córdoba, Argentina.

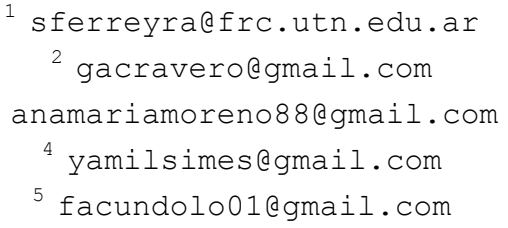

* Departamento Ingeniería Electrónica, Facultad Regional Córdoba Universidad Tecnológica Nacional, Maestro López esq. Cruz Roja Argentina. Ciudad Universitaria Córdoba, Argentina.

${ }^{\S}$ Departamento Materias Básicas, Facultad Regional Córdoba Universidad Tecnológica Nacional, Maestro López esq. Cruz Roja Argentina. Ciudad Universitaria Córdoba, Argentina

Recibido: 07/12/18; Aceptado: 28/03/19

\begin{abstract}
Acoustics is an intrinsically interdisciplinary science that has changed the way it has been taught in recent decades. Currently in higher education its teaching is varied and even opposed, applying methods such as theoreticalpractical lectures to problem-based learning where the student is the main protagonist of their own learning, since the inquiry conducted by the student leads the process. At the same time, the new paradigms of higher education prioritize students research skills. This document describes two examples of guided research, applied pedagogical innovation in the subject Fundamentals of Acoustics and Electroacoustic of the National Technological University, showing favourable results in the learning process.
\end{abstract}

Keywords: acoustics; teaching for understanding; research skills; guided research.

Resumen- La acústica es una ciencia intrínsecamente interdisciplinaria que ha modificado su forma de enseñanza en las últimas décadas. Actualmente en la educación superior su enseñanza resulta variada y aún contrapuesta, aplicando métodos como clases magistrales teórico-prácticas hasta el aprendizaje basado en problemas donde el estudiante es protagonista fundamental de su propio aprendizaje, ya que la indagación realizada por el alumno lidera dicho proceso. Al mismo tiempo, los nuevos paradigmas de la educación superior priorizan las habilidades de investigación de los estudiantes. Este documento describe dos ejemplos de investigación guiada, innovación pedagógica aplicada en la asignatura Fundamentos de Acústica y Electroacústica de la Universidad Tecnológica Nacional, evidenciando resultados favorables en el proceso de aprendizaje.

Palabras clave: acústica; enseñanza para la comprensión; habilidades de investigación; investigación guiada.

\section{INTRODUCCIÓN}

En la actualidad los vertiginosos avances en ciencia y tecnología están configurando un nuevo orden social basado en el conocimiento y en las capacidades de transferencia [1]. En esta sociedad del conocimiento, dichos factores junto con la tecnología se convierten en el motor de desarrollo social y económico de las comunidades. El contexto laboral presenta características cambiantes, las profesiones ya no se encuentran claramente definidas, la estabilidad de los puestos de trabajo se ve amenazada y la interdisciplinariedad es una demanda creciente [2].

El panorama descripto presenta grandes desafíos para la Educación Superior, principalmente para afrontar cambios dando respuesta a demandas actuales. En su informe para la UNESCO de la Comisión Internacional sobre la Educación para el Siglo XXI titulado "La educación encierra un tesoro" específicamente en la sección "Los cuatro pilares de la educación" Delors [3] expresa que los propósitos de la educación deben enfocarse a las acciones concretas de "Aprender a: conocer, hacer, convivir y ser". Señalando que para alcanzar este propósito, se torna imprescindible un cambio en las prácticas docentes. Este cambio, radica principalmente en enfocar dichas prácticas a un nuevo paradigma de enseñanza, centrado en el aprendizaje. En este nuevo escenario, el estudiante debe ocupar el lugar central, como "sujeto que aprende". Los docentes adquieren un nuevo rol, intentando dejar atrás el clásico modelo de profesor que concentra la información y la transfiere. En este nuevo rol la principal tarea de los docentes es ayudar a aprender, para esto, es necesario reflexionar sobre qué acciones llevar a cabo para facilitar el aprendizaje. 
Por lo expuesto, el cuerpo docente debe incorporar acciones pedagógicas que promuevan esta condición activa en los estudiantes, generando oportunidades para que el aprendizaje ocurra y a la vez estar preparados para corregir los errores a tiempo. El primer paso hacia este cambio de paradigma, comienza haciendo una revisión sobre que entendemos por comprensión desde nuestro rol docente, ya que las prácticas que se implementen tienen una relación directa con esta noción. En general, por herencia, tenemos incorporada una visión conductista del aprendizaje, creemos que la comprensión se da cuando el estudiante genera una representación o modelo mental de lo aprendido. La confianza sobre el éxito del proceso (aprendizaje) se da cuando interrogamos a los estudiantes y estos son capaces de reproducir dicha representación o modelo. Por ejemplo: al pedir a los estudiantes que calculen la velocidad del sonido, ellos pueden responder la pregunta aún sin comprender. Es decir no necesariamente son capaces de encontrar ejemplos o preguntas inteligentemente relacionadas al tema y su experiencia cotidiana, lo que asegura la comprensión. Perkins [4] señala que la comprensión "es la habilidad de pensar y actuar con flexibilidad a partir de lo que uno sabe". En función de esta concepción del significado de comprensión, surge el marco teórico que da origen a la enseñanza para la comprensión. En este, se plantean cuatro interrogantes clave, ¿Qué tópicos vale la pena comprender?, ¿Qué aspectos de esos tópicos deben ser comprendidos?, ¿Cómo podemos promover la comprensión? y ¿Cómo podemos saber que los estudiantes comprenden? En respuesta a estas preguntas el Proyecto Cero de la Escuela de Educación de la Universidad de Harvard sobre Enseñanza para la Comprensión (EpC) divide las respuestas de la siguiente forma:

$\checkmark \quad$ El Hilo Conductor, representa el eje central de la comprensión a lo largo del curso.

$\checkmark \quad$ Los Tópicos Generadores, son conceptos y teorías asociadas al hilo conductor que los estudiantes comprenderán.

$\checkmark$ Las Metas de Comprensión, enuncian explícitamente y de manera específica lo que se espera que los alumnos lleguen a comprender.

$\checkmark$ Desempeños de Comprensión, representan actividades para promover la comprensión. Requieren que los estudiantes usen el conocimiento en nuevas situaciones, ayudan tanto a construir la comprensión como a poder demostrarla.

$\checkmark$ Evaluación Continua, basados en la idea de que comprender es un proceso, se incorpora la evaluación continua, de esta manera los estudiantes tienen referencia de la evolución sobre sus desempeños de comprensión y así la posibilidad de mejorarlos. Por su parte el docente debe ajustar las actividades en función de los resultados obtenidos en el proceso de evaluación continua.

Una estrategia que se utiliza en el marco de la EpC, es el Aprendizaje Basado en Problemas (ABP). Esta estrategia se puede pensar como el medio para que los estudiantes adquieran los conocimientos aplicándolos en la solución de un problema real, de esta forma es posible evitar la típica "clase magistral", Barrows [5] define al ABP como "un método de aprendizaje basado en el principio de usar problemas como punto de partida para la adquisición e integración de los nuevos conocimientos".

De la Torre [6] plantea que la tarea docente implica un profesional competente no solo en el domino del contenido formativo sino también debe ser capaz de proponer mejoras a través de estrategias didácticas que motiven a sus estudiantes a aprender.

Finalmente, mencionar que las estrategias elegidas para avanzar hacia la comprensión, dependen también de la respuesta que se obtiene en los estudiantes y si en algún momento notamos que una clase magistral resulta un medio eficaz para favorecer el aprendizaje no debemos renunciar por completo a ella [7].

Las propuestas curriculares basadas en un Modelo de Aprendizaje por Competencias, consideran un conjunto de conocimientos, habilidades y actitudes que nos permiten adaptarnos a la realidad cambiante y desempeñarnos en ella de manera eficiente [8]. Un rasgo esencial de las competencias es la relación entre la teoría y la práctica, en el sentido de proveer tanto de habilidades como de actitudes frente al conocimiento y el contexto propio del campo disciplinar del cual surge o en el que se desarrolla [9].

El Aprendizaje Basado en Proyectos (ABPy) [10], es un claro ejemplo en este sentido y en consecuencia, la propuesta de enseñanza que se diseñe y planifique tiene que estar de acuerdo a las competencias que se pretenden desarrollar. El mencionado modelo se centra en que los estudiantes desempeñan el rol de profesionales basando su experiencia de aprendizaje a situaciones concretas de trabajo conectándolo con la realidad laboral [11].

En el ABPy el estudiante consigue alcanzar la autonomía progresiva en la toma de decisiones sobre los objetivos del trabajo que él mismo propuso, fortaleciendo la construcción significativa de los nuevos conocimientos que va adquiriendo tanto desde la adopción de destrezas y habilidades propias de esa área cognitiva como de las actitudes que entran en juego en la consecución de las proyecciones del proyecto.

En este trabajo, en primer lugar, intentaremos indicar la relevancia que adquiere la formación en investigación como estrategia pedagógica, en pos de favorecer el cambio de paradigma antes mencionado. Entendiendo la formación en investigación como "la enseñanza a través de la investigación" y resaltando la investigación como una estrategia didáctica o como "docencia investigativa" [12].

En segundo lugar, remarcar que el término Investigación Guiada se utiliza para referirse a la formación en investigación como estrategia pedagógica implementada, que contempla los criterios establecidos en $\mathrm{EpC}$, con aspectos del ABP y ABPy. También puede asociarse con algunos aspectos de la estrategia conocida como "aprendizaje por indagación" [13].

La Investigación Guiada se desarrolla desde hace 8 años de manera ininterrumpida en el contexto del espacio curricular Fundamentos de Acústica y Electroacústica, de la Facultad Regional Córdoba, Universidad Tecnológica Nacional. Se pretende que los estudiantes actúen de manera idónea ante una situación profesional, desde un enfoque interdisciplinario, por lo cual se establecen en la asignatura nuevos criterios de evaluación que prioricen el desempeño 
en las condiciones reales de trabajo y no sólo en el cumplimiento formal de objetivos curriculares tradicionales.

\section{MARco Institucional}

La Universidad Tecnológica Nacional (UTN) fue fundada en 1959 para crear, preservar y transmitir el conocimiento cultural universal y técnico en el campo de la tecnología. La UTN presenta dos las características que la distinguen del resto de las instituciones del sistema universitario argentino: a) es la única universidad del país que tiene a la ingeniería como prioridad en su oferta académica, b) posee carácter federal, por incluir a todas las regiones de Argentina a través de sus 33 sedes (Rectorado, 30 Facultades Regionales, 1 Instituto Nacional Superior y 1 Centro Tecnológico de Desarrollo Regional). Esto se traduce en un permanente e íntimo vínculo con los sistemas productivos regionales que promueven un fructífero intercambio académico nacional. Anualmente, la UTN aporta al sistema productivo nacional más del $35 \%$ de graduados en ingenierías. Por otra parte, debido a su presencia en toda la extensión geográfica del país deriva su amplia capacidad de estudiantes, más de 85000, lo cual representa más del $40 \%$ de los estudiantes de ingeniería del país. Actualmente posee 15 carreras de ingenierías, 2 licenciaturas, 55 tecnicaturas superiores, 21 ciclos de licenciaturas, 10 doctorados, 21 maestrías, 28 especializaciones y 55 cursos de actualización. En referencia a dependencias de investigación científicotécnica posee 33 Centros y 66 Grupos de investigación, desarrollo y transferencia, donde se desempeñan más de 1800 docentes investigadores, junto a estudiantes de posgrado y grado.

La carrera de Ingeniería en Electrónica de la Facultad Regional Córdoba, cuenta con 37 asignaturas obligatorias organizadas en 5 niveles, 11 asignaturas electivas de especialización en el sexto nivel (el estudiante debe cursar y aprobar un mínimo de tres asignaturas electivas), una práctica profesional supervisada (superior a $200 \mathrm{Hs}$, con supervisión de un docente), y desarrollar un proyecto final integrador para alcanzar el título de ingeniero en electrónica. La carrera ha sido acreditada por CONEAU, por el término de 6 años (Res. No 485/13) y por MEXA (Acreditación MERCOSUR-ARCUSUR) por término de 6 años (Res. $N^{\circ}$ 479/13).

Fundamentos de Acústica y Electroacústica (FAyE), asignatura única en su tipo dentro de la UTN, es un espacio curricular electivo del sexto nivel $\left(11^{\text {vo }}\right.$ cuatrimestre) de la carrera Ingeniería en Electrónica vigente en el actual plan de estudio y con dictado ininterrumpido desde el año lectivo 2011. Para ser alumno de la mencionada asignatura, el estudiante previamente ha cursado disciplinas básicas como, álgebra, cálculo diferencial e integral, química, física, estadística, informática, entre otras, como también disciplinas específicas de ingeniería en electrónica como señales y sistemas, teoría de circuitos, técnicas digitales, electrónica aplicada, sistemas de comunicaciones, electrónica de potencia, sistemas de control, medias electrónicas, tecnología electrónica, entre otras. Por lo cual, es en dicha instancia final donde el estudiante inicia un trayecto de especialización en la temática que desee a través de las asignaturas electivas.

Cabe destacar que por lo general, los estudiantes se inician formalmente en Acústica al cursar esta asignatura, ya que durante la carrera han adquirido principalmente habilidades y capacidades de análisis, diseño y desarrollo electrónico que les permiten fácilmente generar soluciones técnicas innovadoras para múltiples y variadas situaciones de la vida que involucran diversos tipos de tecnologías. Por lo cual, la formación básica en sonido y procesamiento de señales de audio obtenida durante la carrera previo al cursado de esta asignatura, se convierte en el punto de partida del estudiante hacia la comprensión de esta ciencia interdisciplinaria, donde confluyen conocimientos de física, psicología, música, ingeniería, medicina, arquitectura, entre otros.

La Acústica es una ciencia inherentemente interdisciplinaria, cuyo objeto de estudio es el sonido. El sonido puede ser interpretado de una manera física, como oscilaciones de presión o bien se lo puede asociar a la percepción auditiva que generan estas oscilaciones de presión en una persona [14]. Una Investigación Interdisciplinaria implica una forma abordar problema generado por un sistema complejo. En esta forma de trabajo se analiza la problemática desde distintos enfoques de manera conjunta, de esta manera la visión de cada especialista se integra e interactúa en forma dinámica con el resto del equipo. A partir de la naturaleza dual en la definición del objeto de estudio, los problemas de investigación en acústica requieren de un abordaje interdisciplinario.

\section{EXPERIENCIA EDUCATIVA}

FAyE está organizada en dos partes, la primera es una introducción a la acústica, donde se abordan cinco temáticas fundamentales: a) la acústica como ciencia interdisciplinaria, b) acústica física, c) fonación, audición y percepción sonora, d) acústica arquitectónica, e) metrología acústica. En la segunda, se estudian los componentes que intervienen diversos sistemas electroacústicos, poniendo énfasis en el diseño e implementación electrónica, siendo sus cinco temáticas: f) electroacústica, g) micrófonos, h) fuentes sonoras artificiales y naturales, i) procesadores de audio, j) sistemas de sonido para espacios cerrados y abiertos.

En relación a los objetivos, el cursado de esta asignatura le permite al alumno adquirir nuevos conocimientos sobre Acústica e integrar aquellos que fueron vistos durante la carrera en materias afines. Estos conocimientos le permitirán por un lado, iniciarse profesionalmente como Ingeniero Electrónico con orientación en Acústica y por el otro, podrá continuar sus estudios de posgrado en esta disciplina en diferentes universidades del país e insertarse en el Sistema de Ciencia y Técnica, realizando investigación y desarrollo tecnológico.

Acorde a estos objetivos, y dado que la asignatura se desarrolla en 16 semanas de clases, nuestra propuesta metodológica es la siguiente:

En cada semana se dictan un total de 6 horas distribuidas en dos clases. La modalidad de cada una es teórica-práctica donde se introducen conceptos y modelos acústicos desde los más simples a los más complejos. También se trabaja con diferentes programas de medición, análisis y simulación acústica de distribución libre como REW, Audacity, OCTAVE, SciLAb, ELMER, BassBoxPro y versiones demo 
como ODEON, DIRAC, CADNA y COMSOL. En todos los casos apunta a que el estudiante comprenda y desarrolle habilidades sobre cómo utilizar la herramienta e interpretar los resultados del programa con el fin de resolver los problemas planteados. De esta manera el docente adquiere un rol de mediador entre la interpretación de los contenidos y las ventajas del uso de ciertas herramientas y habilidades de desempeño, siempre acordes a las propuestas de solución presentadas por los estudiantes. Así, el docente facilitador se torna como tutor de la toma de esas decisiones, promoviendo a una actitud reflexiva en sus estudiantes.

El principal propósito de cada clase es presentar distintos modelos acústicos, métodos y técnicas de análisis para que el estudiante, al terminar el curso, haya desarrollado un nutrido conjunto de habilidades y un importante bagaje de herramientas en cuanto al análisis de datos estimulando su capacidad crítica de identificar el mejor modelo, método y técnica que se ajuste al funcionamiento de un sistema. En lo relativo a la evaluación y acreditación del espacio curricular, el mismo posee tres instancias de evaluaciones parciales. Las dos primeras evaluaciones son de índole individual, formativa y acumulativa y la tercera evaluación presenta dos instancias una grupal y otra individual, y se construye durante el desarrollo de la asignatura.

\section{A. Relato de la Experiencia}

Desde su implementación en 2011 las clases son teóricas-prácticas, haciéndose hincapié en la relación de los contenidos conjuntamente con las habilidades prácticas que éstos representan en el aprendizaje de los estudiantes. El proceso de aprendizaje se logra introduciendo diversos temas, realizando análisis y propiciando el debate más la realización de experiencias guiadas simples en el aula y complejas en el Laboratorio de Ensayos Acústicos del CINTRA, CONICET-UTN

Como se mencionara, se toman dos evaluaciones parciales (semi-estructuradas), de carácter teórico-prácticas, que se basan en los contenidos y experiencias realizadas en la primera etapa y segunda etapa de la asignatura.

El tercer dispositivo de aprendizaje y evaluación es un trabajo grupal de investigación guiada por el docente el cual coadyuva a los estudiantes a generar un trabajo original sobre una temática de Acústica seleccionada por ellos. Este dispositivo con características de proceso y construcción grupal se documenta a través de la confección de un informe técnico cumplimentando requisitos específicos de formato y estilo propuestos por la cátedra (norma para confección de informes técnicos de la cátedra FAyE) el cual es sometido a revisión por parte del equipo docente para su publicación electrónica en el sitio web de la asignatura. Esta innovación pedagógica se convierte en un instrumento de evaluación por competencias de trabajo grupal e individual el cual es evaluado en dos instancias: a) escrita y b) oral a través de una ponencia de 15 minutos y 5 minutos de preguntas realizadas por el docente y pares alumnos.

Además, los alumnos realizan 4 Trabajos Prácticos (TP), que se convierten en instrumentos de evaluación basados en competencias adquiridas, donde los estudiantes tienen que enfrentar situaciones problemáticas específicas abordándola desde aspectos teóricos y utilizando metodologías que den respuesta en forma holística al problema planteado.
También, en este dispositivo el docente establece un marco de referencia "guía" para que el estudiante desarrolle sus capacidades.

Finalmente, en carácter de cierre se realiza un intercambio práctico vivencial a través de una visita a organizaciones y/o espacios donde la acústica posee un rol singular tal como: estudios de grabación musical, estudios de radiodifusión, auditorios, salas de concierto, teatros, bibliotecas, laboratorios, industrias, entre otros. En estas visitas, los estudiantes realizan una actividad de cierre de contenidos "in situ" y también intercambian conocimientos adquiridos y experiencias vividas con diversos actores como músicos, ingenieros de sonido, arquitectos, médicos, fonoaudiólogos, psicólogos, entre otros.

\section{B. Investigación Guiada}

Como se ha mencionado, el tercer dispositivo de evaluación pretende, dentro de la formación en competencias reservadas al desempeño profesional de los futuros ingenieros en electrónica, fomentar la investigación interdisciplinaria en acústica, la innovación tecnológica y el desarrollo de tecnologías que permitan generar soluciones integrales a diversos tipos de problemáticas acústicas. La metodología didáctica que mejor se ajusta al objetivo, es el Aprendizaje Basado en Proyectos dado que, por su característica, permite acompañar la elaboración del mismo durante el desarrollo de la asignatura. El modo en que se desarrolla es el siguiente: durante la primera semana de clase se definen los lineamientos generales de la realización del trabajo/proyecto de investigación y elaboración del correspondiente informe técnico, como también el proceso de revisión al cual será sometido para su publicación. A tal fin, el alumno, dispone del cronograma completo de actividades programadas en la asignatura, las fechas de todas las evaluaciones y entregas de trabajos prácticos, como también una guía para establecer el tipo de trabajo a realizar (modelado, simulación, experimentación, validación, comparación, entre otros) y los requisitos de formato y estilo del informe técnico, donde dependiendo del tipo de trabajo la estructura podría contemplar:

- Título

- Autores

- Filiación

- Resumen (en español e inglés)

- Introducción

- Objetivos

- Antecedentes

- Conceptos y Términos

- Normas

- Hipótesis

- Metodología

- Arreglo experimental, Métodos aplicados

- Resultados

- Discusión

- Conclusiones

- Sumario

- Trabajo a futuro

- Agradecimientos

- Referencias

- Datos biográficos de los autores 
Se explica a los estudiantes que dicho trabajo debe ser realizado de modo colaborativo en grupos de 2 a 4 durante el cursado la asignatura y se presentan los criterios de evaluación.

Luego del primer parcial, los grupos conformados han definido el tema seleccionado, y de manera continua todas las clases se destinan los últimos 15 minutos para trabajar en los proyectos donde el docente actúa como guía y facilitador. Durante todo el cursado de la asignatura los estudiantes utilizan los horarios de consultas para analizar críticamente $\mathrm{y}$, de forma conjunta con el docente, la progresión de sus aprendizajes. Se les propone que actúen como investigadores y profesionales profundizando el tema elegido, consultando entre pares y a otros docentes, solicitando información a especialistas y expertos nacionales e internacionales, realizando experiencias y simulaciones, con el fin de que puedan organizar, delimitar y planificar paso a paso todas las actividades que requieran para la ejecución del proyecto.

Para que el aprendizaje se lleve a cabo, los alumnos necesitan superar distintas dificultades y equívocos durante el proceso. Esto implica para ellos un aprendizaje de autorregulación ya que transforman sus habilidades mentales en habilidades académicas [15]. Para alcanzar el producto final los estudiantes se enfrentan a situaciones que se convierten en verdaderos problemas a resolver: identificar y caracterizar fuentes acústicas, e investigan sobre la problemática inherente a la Acústica en la cual se sitúan, plantean objetivos y elaboran preguntas de investigación acordes a estos, establecen hipótesis, y plantean posibles experimentaciones para validar resultados, proponen modelos, y resuelven las formas de medir, calcular incertidumbres y procesar datos. Otro aspecto importante que tienen que sortear los estudiantes, es que esta dinámica de trabajo los obliga a debatir, acordar, tomar decisiones y justificarlas, volver sobre los objetivos y redefinirlos y hacer nuevas preguntas [16].

Las competencias específicas que esta propuesta de enseñanza apunta a fomentar son: la planificación, la creatividad, la argumentación, la indagación, la autonomía, el trabajo en equipo y la expresión oral y escrita.

En el cronograma de la materia se contempla que, en las cuatro últimas clases, luego del segundo parcial, se realice la presentación escrita y oral de los trabajos con una duración de máxima de 20 minutos distribuidos en $15^{\prime}$ para la exposición del proyecto y 5 ' para responder preguntas o dudas de compañeros y docentes. Es una actividad de cierre como culminación de la asignatura donde se sociabiliza y se comparte la tarea elaborada por cada equipo y se lo somete al análisis reflexivo y crítico entre pares y docente.

Luego, son evaluados utilizando dos rúbricas públicas; una para evaluación grupal e informe técnico y otra para evaluación individual enfocada a la ponencia oral. Algunos de los ítems más relevantes que se evalúan a nivel grupal son: presentación en tiempo y forma, originalidad del trabajo (enfoque y profundidad), coherencia interna del trabajo y las referencias utilizadas. En el caso de la rúbrica individual los ítems buscan evaluar si la terminología empleada es correcta para los conceptos trabajados, la oratoria y forma de expresar los conceptos son acordes al trabajo realizado, el material presentado en las diapositivas/videos/audios es significativo al trabajo y permite su correcta visualización/audición, entre otros. La calificación que se puede alcanzar en estos ítems es: Alto (10-9-8), Medio (7-6), Bajo (5-4), Muy Bajo (3-2-1).

La evaluación oral se completa el mismo día de la ponencia indicando el nivel correspondiente y realizando una devolución personal a cada estudiante. Esta etapa de la evaluación, es realizada por el docente en forma conjunta con los estudiantes convirtiéndose en una oportunidad de autoevaluación y en una instancia de retroalimentación sobre el rendimiento colectivo e individual. Luego, se establece la nota final al revisar y evaluar los trabajos en su versión escrita y se promedia esta calificación con la lograda individualmente.

\section{RESULTADOS OBTENIDOS}

Desde el primer año lectivo de la asignatura la aplicación de esta metodología propuesta favoreció que el estudiante integre teoría y práctica de manera intuitiva, fácil y con una dinámica diferente, despertando en los estudiantes de ingeniería sus condiciones como investigador tecnológico en el campo de la Acústica.

Del mismo modo la actividad implica en sí misma una discusión tanto interna a los grupos de trabajo de estudiantes, como externa a los estudiantes de los demás grupos. En la primera, se consensuan situaciones, se eligen diferentes modelos y métodos para aplicarse a diferentes sistemas acústicos. Por otra parte, en la segunda, se exponen los resultados del trabajo a sus pares y se defienden cada una de las elecciones realizadas por cada grupo. Esta nueva dinámica genera un proceso de aprendizaje diferente, donde la colaboración y el pensamiento crítico adquieren nuevas dimensiones.

A fines ilustrativos se presenta un resumen de dos casos de trabajos de investigación guiada, ambos con altas calificaciones grupales e individuales, donde se presenta la estructura del trabajo y la coherencia de contenidos. Cabe destacar que el objetivo del primer trabajo fue realizar un análisis de las características físicas de materiales absorbentes sonoros porosos de tipo resistivo; mientras que del segundo, fue analizar y describir la implementación de control activo de ruido en auriculares:

\section{Caso A:}

Título: Características físicas de materiales absorbentes sonoros porosos.

Autores: J. Castillo y A. Costa

Fecha de publicación: Mayo de 2012

Estructura del trabajo:

1. Introducción

2. Principio de funcionamiento

3. Propiedades físicas de materiales absorbentes porosos.

3.1 Propiedades microscópicas

3.1.1 Resistividad al flujo

3.1.2 Porosidad

3.2 Propiedades macroscópicas

3.2.1 Factor de forma y dimensiones

3.2.2 Tortuosidad

4. Parámetros

4.1 Coeficiente de absorción sonora

4.2 Coeficiente de reducción acústica 
5. Variación del coeficiente de absorción sonora del material poroso en función de su posición y características.

5.1 Efecto del espesor

5.2 Efecto de la porosidad

5.3 Efecto de la densidad

5.4 Efecto de la distancia a pared rígida

5.5 Efecto de la geometría superficial

6. Tipos de absortores porosos

6.1 Fibras

6.1.1 Fibras de kenaf

6.1.2 Fibra de poliéster

6.1.3 Lana mineral o de roca

6.1.4 Fibra de vidrio

6.2 Polímeros

6.2.1 Melaminas

7. Modelización numérica utilizando FEM

8. Conclusiones

9. Referencias

10. Datos biográficos

\section{Caso B:}

Título: Auriculares con control activo de ruido.

Autores: L. Budde, M. Zannier y G. Alonso

Fecha de publicación: Junio de 2012

Estructura del trabajo:

1. Introducción

2. Control de ruido

2.1 Control pasivo de ruido

2.2 Control activo de ruido

3. Sistemas de control activo de ruido

3.1 Sistemas de control activo de ruido prealimentado

3.2 Sistemas de control activo de ruido realimentados

4. Control inverso adaptativo

4.1 Algoritmo de mínimos cuadrados

4.2 Algoritmo LMS con filtro X

5. Factores principales del control activo de ruido

6. Selección de transductores

6.1 Dinámica lineal

6.2 Dinámica no lineal

6.3 Ruido propio

7. Aplicaciones prácticas

8. Comparación de modelos comerciales

9. Conclusiones

10. Referencias

11. Datos biográficos

Los resultados de ambos casos superaron las expectativas, los trabajos alcanzaron los objetivos planteados por los estudiantes y el equipo docente, sus ponencias recibieron numerosas consultas entre pares y despertaron interés en continuar investigaciones en los estudiantes. Además fueron citados en artículos posteriores presentados en congresos nacionales y utilizados en proyectos finales de grado de la carrera de ingeniería en electrónica de la UNC y UTN.

Seguidamente consideramos significativo recuperar las opiniones más recurrentes de los estudiantes que manifiestan:

"lo más difícil fue decidir el tema de investigación, la organización del grupo, la búsqueda de artículos científicos (papers) sobre el tema, la redacción del informe técnico llevo mucho tiempo y corrección" (FAyE 6R1, 2011) "el trabajo nos sirvió porque pudimos pensar la materia como un todo, uniendo lo teórico con lo práctico" (FAyE 6R1, 2012)

"la ponencia oral en tiempo estipulado fue todo un desafío, sin embargo el grupo cumplió el objetivo de no penalizar, fue una experiencia muy enriquecedora" (FAyE 6R1, 2013)

"aprendimos temas nuevos, trabajamos en grupos y generamos soluciones a problemas acústicos" (FAyE 6R1, 2014)

"aprendimos que podemos diseñar e implementar nuestros propios sistemas acústicos de medición, elaboramos nuestra propia experiencia en función de nuestros intereses y aplicamos conceptos de acústica en el proyecto final de la carrera" (FAyE 6R1, 2015)

“aprendimos métodos de procesamiento digital de señales acústicas que pueden ser aplicados a múltiples problemas (FAyE 6R1, 2016)

"los trabajos prácticos fueron desafiantes e interesantes, la visita al estudio de grabación y el intercambio de experiencias fue lo mejor de la materia" (FAyE 6R1, 2017)

"la acústica es una ciencia interdisciplinaria que seguramente seguiré estudiando, fue una muy buena experiencia el trabajo de investigación y su presentación oral" (FAyE 6R1, 2018)

Por otra parte, cabe mencionar que la asignatura en estos ocho años tiene un promedio de inscriptos superior a 20 alumnos. A partir del ciclo lectivo 2018, y según el reglamento vigente de la Cátedra en cumplimiento con la Ord. UTN N ${ }^{\circ} 1549$, que establece la aprobación directa de las asignaturas por primera |ves en la historia de la UTN, la condición final de los estudiantes del ciclo lectivo 2018 fue del $100 \%$ aprobado en forma directa, mientras que en los ciclos lectivos de años anteriores en promedio el $82 \%$ regularizaban la asignatura y a su vez promocionaban (pasaban a coloquio) el 63\%.

\section{CONCLUSIÓN}

El cambio de paradigma de la educación superior está dando evidencias significativas en la calidad del aprendizaje de los alumnos. Las propuestas curriculares basadas en un modelo de aprendizaje por competencias están demostrando que se logran mejores resultados que con el método tradicional de clases magistrales.

El aprendizaje basado en proyecto, sin lugar a dudas, es una estrategia didáctica valiosa e interesante donde se involucran simultáneamente varias competencias que el estudiante pone en acción para la apropiación de contenidos, así como en la puesta en ejercicio de otras habilidades que son imprescindibles en su formación profesional [16].

$\mathrm{El}$ instrumento pedagógico presentado como "investigación guiada", articula además criterios del ABPy con los del ABP, y por su forma de desarrollo resulta una óptima actividad complementaria para el dictado de un 
curso de materias de especialización, donde el resultado se obtiene hacia el final del cursado. Es por lo tanto, un trabajo longitudinal que en su elaboración, acompaña al desarrollo de la asignatura, lo cual implica un proceso de aprendizaje diferente y significativo, ya que los estudiantes revisan su trabajo constantemente, reflexionan sobre él, incorporan nuevas ideas descartando otras y efectuando cambios convenientes.

La elaboración de un cuadro de evaluación claramente establecido y comunicado a los alumnos, permite tener una visión integrada y más precisa para evaluar el desempeño de los alumnos en relación a las competencias que involucra.

Finalmente, consideramos que la modalidad implementada en el curso de esta asignatura electiva está colaborando en la formación de futuros ingenieros en lo respectivo a las capacidades de los mismos para realizar actividades profesionales y de investigación para resolver problemas creando acciones productivas y colaborativas en el marco de la actual "sociedad del conocimiento".

\section{AGRADECIMIENTOS}

Los autores desean agradecer al Ing. Mario R. Serra, Lic. Ester C. Biassoni, Lic. Ana Verzini, Dra. Claudia Arias, Ing. Oscar A. Ramos, An. Sis. Aldo H. Ortiz Skarp y Ing. Carlos Frazzoni, miembros fundadores del Centro de Investigación y Transferencia en Acústica, CINTRA quienes formaron a los actuales docentes-investigadores del CINTRA que se desempeñan en diversas cátedras de la UTN y la UNC.

\section{REFERENCIAS}

[1] J.R. Bello. "Los nuevos paradigmas para la formación de los ingenieros". Cuadernos Unimetanos, (29), 23-28. 2012.

[2] J. G. Mora, "La necesidad del cambio educativo para la sociedad del conocimiento". Revista Iberoamericana de educación, 35(2), 13-37. 2004.

[3] J. Delors, Los cuatro pilares de la educación en La educación encierra un tesoro. Informe a la UNESCO de la Comisión internacional sobre la educación para el siglo XXI, Madrid, España: Santillana/UNESCO. pp. 91-103. 1996.

[4] D. Perkins \& C. Unger. La enseñanza para la comprensión. 1999.

[5] H.S. Barrows, "A taxonomy of problem-based learning methods". Medical education, vol. 20 (6), 481-486. 1986.

[6] S. De la Torre, "La Universidad que queremos. Estrategias creativas en el aula universitaria". Revista digital Universitaria Vol. 10 (12) 2009.

[7] P. Meirieu, A mitad de recorrido: por una verdadera "revolución copernicana" en pedagogía. Frankenstein educador. Alemania. 2000

[8] M. García Cano, (2008). "La evaluación por competencias en la educación superior". Profesorado. Revista de Currículum y Formación de Profesorado, Vol. 12 (3): 1-16. 2008

[9] J.J.H. Amezola, I.S.P. García, A.R.C. Castellanos, (2008) "Desarrollo curricular por competencias profesionales integrales". Revista Educar, Vol.13, pp. 15-20. 2008

[10] S. Tobón, S., La formación basada en competencias en la educación superior: el enfoque complejo. Ed. Universidad Autónoma de Guadalajara. Mexico. 2008

[11] M. Maldonado Rojas y M. Vasquez Rojas. "Experiencia de desarrollo de un proyecto de aprendizaje colaborativo como estrategia formativa". Educ Med Super, Vol.22, n.1, pp. 1-10. ISSN 0864-2141. 2008

[12] M. Romero Ariza. "El aprendizaje por indagación:¿existen suficientes evidencias sobre sus beneficios en la enseñanza de las ciencias?." Revista Eureka sobre enseñanza y divulgación de las ciencias 4.2 (2017): 286-299

[13] N. Hewitt, \& F. Barrero, La integración de los saberes: una propuesta curricular para la formación en investigación en la educación superior. Psychologia, 6(1), 137-145. 2012.

[14] A. Carrión Isbert, Diseño acústico de espacios arquitectónicos. Ed. UPC, Barcelona, España, 1998.
[15] M. Maldonado Perez, "Aprendizaje basado en proyectos colaborativos. Una experiencia en educ M. Maldonado Perez, "Aprendizaje basado en proyectos colaborativos. Una experiencia en educación superior". Laurus, Vol. 14, Núm. 28: 158-180 Universidad Pedagógica Experimental Libertador Venezuela. 2008.

[16] H.A. Cugno, R. Forestello, A. Mangueaud, "El aprendizaje basado en proyecto aplicado a la asignatura diseño experimental en la carrera de ciencias biológicas de la UNC". Revista Facultad de Ciencias Exactas Físicas y Naturales, vol 5, 2, 83-87, Córdoba. 2018 